\title{
Affinage et qualité du Gruyère de Comté \\ II. Influence de l'affinage sur l'évolution des caractéristiques physico-chimiques des fromages
}

\author{
J.L. BERDAGUE * et R. GRAPPIN \\ avec la collaboration technique de G. DUBOZ \\ I.N.R.A., Station expérimentale laitière, 39800 Poligny, France \\ * Institut Technique du Gruyère
}

\section{Résumé}

Cet article décrit l'évolution moyenne des principales caractéristiques physicochimiques du Gruyère de Comté à différents stades d'affinage. Les valeurs moyennes, à $20 \mathrm{~h}$ et en fin d'affinage, sont respectivement de 5,30 et 5,85 pour le $\mathrm{pH}$, de 62,5 et $66,0 \%$ pour l'extrait sec et, par rapport à l'extrait sec, de 1,54 et $1,46 \%$ pour le calcium et $0,11 \%$ et $1,04 \%$ pour le chlorure de sodium.

La prise en compte des facteurs affinage, fromagerie et saison a permis d'expliquer, suivant les variables, entre 29 et $65 \%$ des variations de composition des fromages au stade coupe.

Par le biais du couple temps-température et du degré d'hygrométrie des caves, les conditions d'affinage influencent essentiellement l'extrait sec et le $\mathrm{pH}$ des fromages. Des teneurs en ammoniac élevées et des apports élevés en sel favorisent la migration du calcium vers la croûte.

Le rapport $\mathrm{Ca} /$ caséine du lait et la teneur en lactate $\mathrm{D}$ du fromage à 20 heures expliquent $35 \%$ des variations du taux de calcium du fromage à 20 heures.

La teneur en chlorure de sodium est essentiellement influencée par les apports effectués en fromagerie et pendant l'affinage en cave chaude, le gain de sel des fromages les plus humides étant le plus important.

Mots clés : Fromage - Gruyère de Comté - pH - Extrait sec - Calcium - Chlorure de sodium.

Ce programme a reçu le soutien financier de la Direction des Industries Agricoles et Alimentaires du Ministère de l'Agriculture (Contrat DIAA $\mathrm{n}^{\circ}$ 81/27) et de l'association Nationale pour la Recherche et la Technologie (Bourse CIFRE de J.L. Berdagué, Convention $\mathbf{n}^{\circ}$ 92/82). 


\section{Summary}

\section{Ripening and quality of Gruyère of Comté cheese \\ II. Influence of the ripening conditions on $\mathrm{pH}$, moisture, calcium and sodium chloride contents of cheese}

This paper describes the average modifications of the main physico-chemical charateristics of Comté cheese during ripening. The mean values at $20 \mathrm{~h}$ and at the end of the ripening period are respectively : 5.35 and 5.85 for $\mathrm{pH} ; 62.5$ and $66.0 \%$ for dry matter ; 1.54 and $1.46 \%$ in dry matter for calcium and 0.11 and $1.04 \%$ in dry matter for $\mathrm{NaCl}$.

The following factors: type of ripening, origin of cheese (cheese plant), and season, explain between 29 and $65 \%$ of the variance of the physico-chemical characteristics of the mature cheese.

Ripening time and temperature, together with the air humidity of the ripening rooms, are the major factors which influence the cheese $\mathrm{pH}$ and moisture. High level of ammonia in the ripening room and addition of salt increase migration towards the rind. The ratio $\mathrm{Ca} /$ Casein of the processing milk and the amount of $\mathrm{D}$ Lactate in cheese at $20 \mathrm{~h}$ explain $35 \%$ of the variation of the calcium content in one-day cheese.

The amount salt is mainly influenced by the quantity of salt added at the cheese plant and during the ripening period in warm room. The $\mathrm{NaCl}$ uptake is closely related to the initial cheese moisture.

Key words : Cheese - Gruyère of Comté - Ripening - pH - Moisture - Calcium Sodium chloride.

\section{Introduction}

Ce travail, qui s'inscrit dans le cadre du programme d'étude sur l'affinage et la qualité du Comté décrit par Grappin et al. (1987), a pour but de mesurer l'évolution des principaux paramètres physico-chimiques des fromages ( $\mathrm{pH}$, extrait sec, teneur en calcium et en chlorure de sodium) au cours de l'affinage.

Le protocole expérimental a permis également de déterminer l'influence des techniques d'affinage des fromages sur ces différents paramètres en fonction de l'origine des fromages (fromagerie) et de la saison de fabrication.

\section{Matériel et méthodes}

\section{A. Protocole expérimental}

Rappelons que les 96 fromages de l'étude représentent 24 fabrications réalisées par 4 fromageries ( $F 1, F 2, F 3$, et $F 4$ ) sur une période de 6 mois (3 mois d'hiver et 3 mois d'été). Au cours de chaque fabrication, 4 fromages ont été obtenus à partir d'une même cuve. Ces 4 fromages, considérés comme étant de composition identique, ont été affinés par 4 affineurs différents (A1, 
A2, A3 et A4). Le schéma expérimental correspond à un plan factoriel dont les niveaux sont répartis comme suit: Fromagerie (4 niveaux), Affinage (4 niveaux), Saison de fabrication ( 2 niveaux), Mois (3 répétitions). Les éléments de caractérisation des différents niveaux des facteurs étudiés sont déjà décrits (GrapPin et al., 1987).

\section{B. Analyses chimiques}

\section{Echantillons}

- Stade de prise des échantillons : chacun des 96 fromages étudiés a été analysé à différents stades de l'affinage. Le $\mathrm{pH}$, l'extrait sec, les teneurs en calcium et en chlorure sont mesurés sur les fromages à $20 \mathrm{~h}$ (stade $\mathrm{F}$ ), à 3 semaines avant affinage en cave chaude (stade A), pendant l'affinage à 9 semaines (stade P) et en fin d'affinage entre 4 et 9 mois (stade C). La teneur en matière grasse a été déterminée au stade $\mathrm{F}$.

- Technique d'échantillonnage : $\mathrm{pH}$, extrait sec au stade $\mathrm{F}$ et matière grasse ont été déterminés sur un mélange de deux sondes cylindriques de 20 $\mathrm{mm}$ de diamètre prélevées en talon et en milieu de rayon. Les autres analyses ont été effectuées sur des sondes prélevées en milieu de rayon.

\section{Méthodes analytiques}

- $\mathrm{pH}$ : mesure, après broyage du fromage, avec une électrode à chlorure de potassium placée en contact avec le broyat tassé dans un godet. Après 30 secondes, l'équilibre est atteint et la mesure du $\mathrm{pH}$ est relevée.

— Extrait sec (ES : en g/100 g de fromage) : Norme FIL 4A (1982).

- Matière grasse (MG : $\mathrm{g} / 100 \mathrm{~g}$ d'extrait $\mathrm{sec}$ ) : méthode butyrométrique de Heiss C, 1962).

- Calcium (Ca: en g/100 g d'extrait sec) : dosage complexométrique selon la technique de Pearce (1977).

- Chlorure ( $\mathrm{NaCl}$ : en $\mathrm{g} / 100 \mathrm{~g}$ d'extrait $\mathrm{sec})$ : mesuré à l'aide d'un chloruremètre (Corning, 926) à électrode soluble d'argent, étalonné par rapport à la technique de mesure des chlorures préconisée par la Norme FIL, 17A (1972).

- Matière azotée (Nx 6,38, exprimée en $\mathrm{g} / 100 \mathrm{~g}$ d'extrait sec) : dosage de l'azote par méthode Kjeldahl (projet de norme FIL E-Doc 214, 1985).

\section{Résultats et discussion}

A. Composition moyenne, variabilité et évolution en cours d'affinage

L'évolution moyenne et la variabilité du $\mathrm{pH}$, de l'extrait sec et des taux de calcium et chlorure de sodium sont représentées sur la figure 1.

- Le pH moyen des fromages varie de 5,30 à 5,85 en cours d'affinage, soit une augmentation de 0,55 unité en cours d'affinage. 


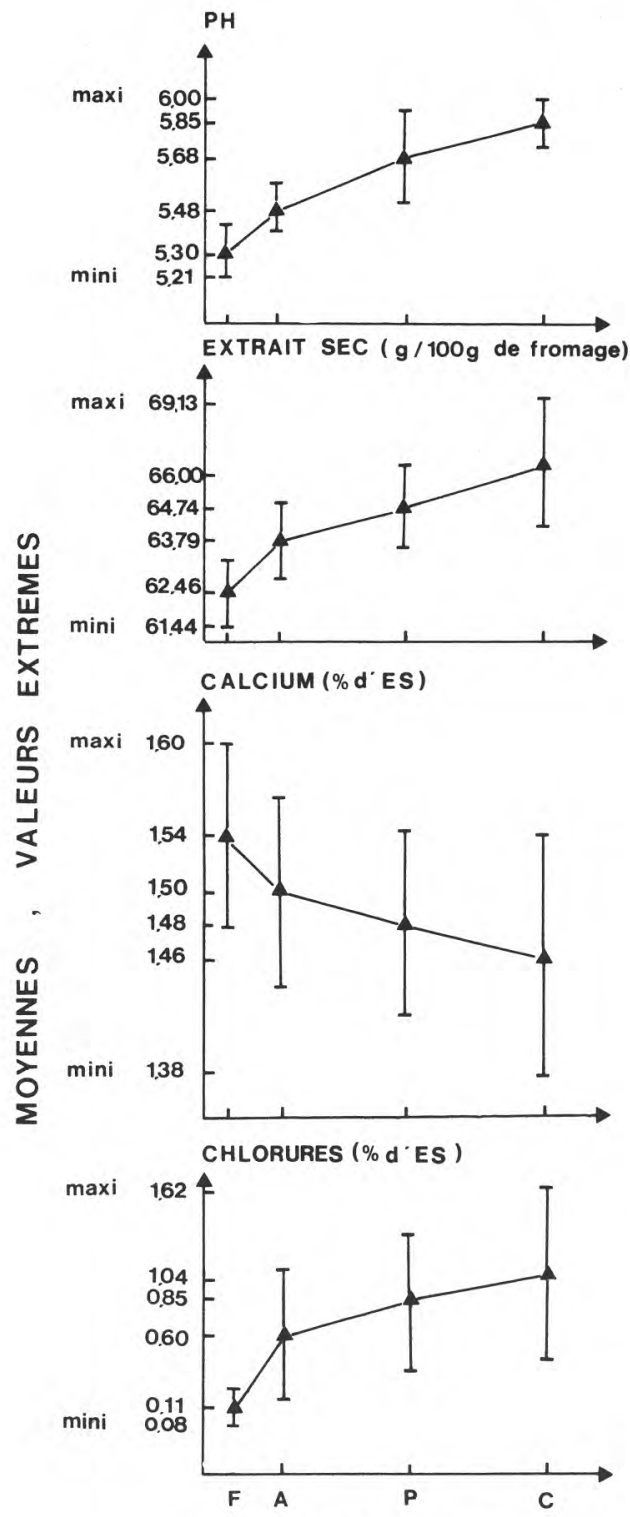

Fig. 1

Evolution au cours de l'affinage des principales caractéristiques physico-chimiques du Gruyère de Comté. Chaque graphique représente la moyenne et l'amplitude de variation ( \pm 2 écart-types) des analyses faites sur les 96 fromages aux différents stades d'affinage.

$F: 20 h ; A: 3$ semaines; $P=6$ semaines ; $C$ : fin d'affinage.

Evolution of the main physico-chemical characteristics of the Gruyère of Comté cheese during ripening. Each graph represents the mean and range of varation $( \pm 2$ standard deviations) of tests carried out on the 96 cheeses at different ripening stages. $F: 20 h ; A: 3 w k ; P: 6 w k ; C$ : end of ripening. 
- L'extrait sec augmente d'environ 4,6\% au cours de l'affinage pour atteindre $66 \%$ en fin d'affinage. Sa variation correspond aux pertes en produits volatils (eau, ammoniac, acides gras et composés volatils) et aux échanges de solutés lors des soins en cave (apport de sel).

- Les teneurs en matière grasse et en protéines des fromages sont en moyenne respectivement de $49,5 \%$ et $43,9 \%$ de l'extrait sec total.

- Entre le fromage à 20 heures et la fin de l'affinage, la teneur moyenne en calcium diminue de $5,5 \%$ en valeur relative pour atteindre la valeur de $1,46 \mathrm{~g} / 100 \mathrm{~g}$ d'extrait $\mathrm{sec}$. Cette perte, liée à une migration du calcium vers la croûte, provient du fait que la partie superficielle du fromage a été éliminée avant analyse.

- La variation du taux de chlorure de sodium est importante à cause du salage en cave d'affinage qui entraîne un gain moyen de 0,93 gramme de sel par $100 \mathrm{~g}$ d'extrait sec.

\section{B. Relations entre variables}

La forte corrélation $(\mathrm{r}=-0,60)$ observée entre le taux de calcium et le taux de chlorure de sodium au stade coupe est en partie liée au déplacement du calcium par le sodium. On constate en effet que les pertes de calcium sont aussi corrélées $(\mathrm{r}=-0,39)$ aux gains de chlorure au cours de l'affinage, malgré des fluctuations très importantes des taux de chlorure entre fromages. Nos résultats sont en accord avec les travaux de GeurTs et al. (1974) sur Gouda et ceux de MAYR (1976) sur Emmental, montrant qu'il peut exister un échange entre le calcium des micelles de caséines et les ions sodium du sel. Les liaisons calcium assurant la structure de la caséine sont détruites, ce qui va entraîner une structure de pâte plus fragile (GeurTs et al., 1980). Dans ce mécanisme ce sont les ions sodium qui sont impliqués directement et non les ions chlorure.

Par ailleurs, on constate que la perte en calcium durant l'affinage est correlée positivement à la perte d'eau dans le fromage $(\mathrm{r}=0,39)$ traduisant une migration puis une accumulation du calcium soluble dans la croûte.

Dans le Camembert, Le Graet et al. (1983), Metche et Fanni (1978), ont observé une migration analogue du calcium vers la surface. Cette migration semble être sous la dépendance de la pénétration du sodium, du gradient de $\mathrm{pH}$ et de la migration de l'eau vers l'extérieur, la flore fongique participant activement à la décalcification de la pâte. Ainsi, la croûte d'un Camembert peut contenir $80 \%$ du calcium total (Ribadeau-Dumas, 1984). En Comté, la concentration du calcium dans la croûte est un phénomène de moindre ampleur, très certainement à cause des dimensions différentes et de la teneur en eau plus faible. Par ailleurs aucun lien n'a pu être mis en évidence avec le $\mathrm{pH}$ du fromage.

\section{Influence des facteurs}

Par analyse de la variance des résultats au stade coupe (tabl. 1), il a été possible de hiérarchiser l'incidence des facteurs (Affinage, Fromagerie, Saison) et de leurs interactions sur les variables analytiques. Toutes les interactions 
TABLEAU I

Valeurs moyennes et écarts types des principales caractéristiques physico-chimiques des fromages en fin d'affinage (exprimées en $\mathrm{g} / 100 \mathrm{~g}$ d'extrait sec), classés en fonction des facteurs affinage, fromagerie et saison

Mean values and standard deviations of the main physico-chemical characteristics of mature cheese (expressed in percent in dry matter), classified according to the factors : ripening conditions, cheese origin (cheese plant) and season

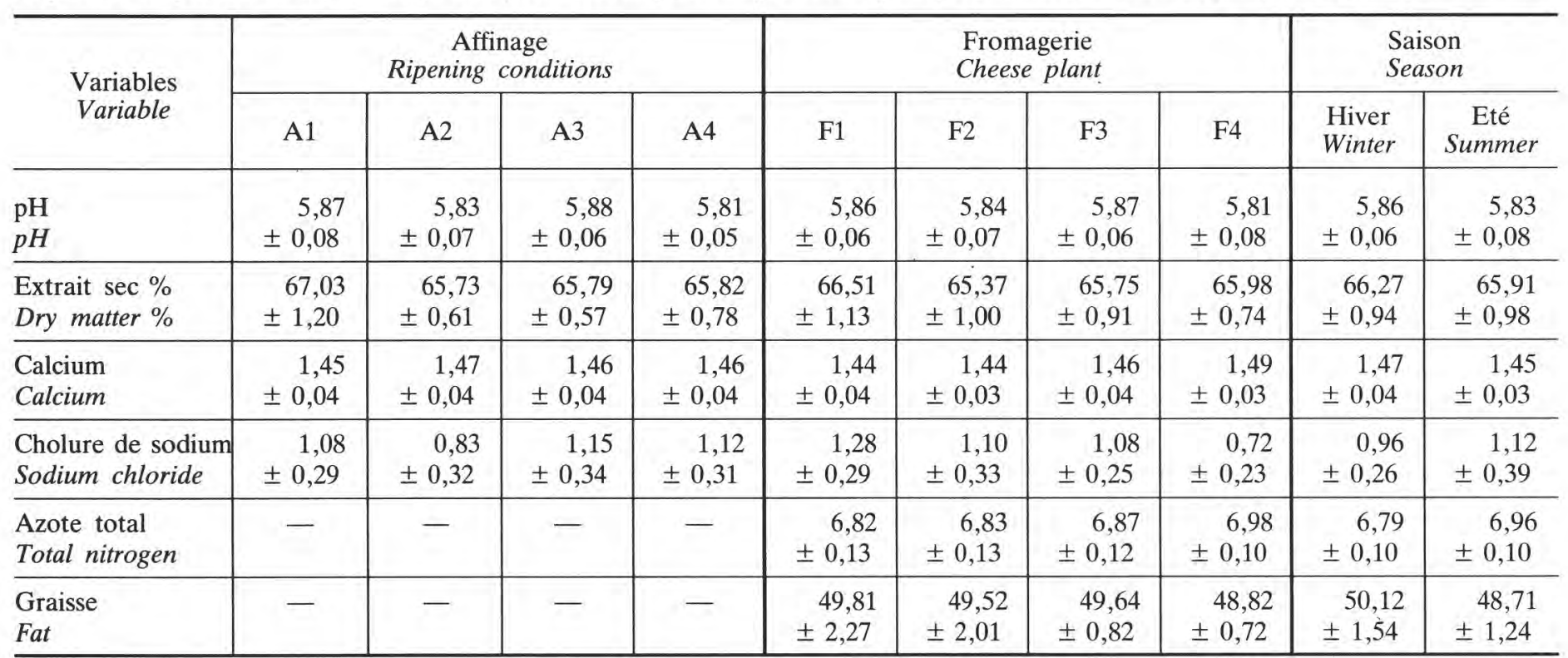


ayant été trouvées non significatives, nous résumons dans le tableau 2 uniquement les pourcentages de la variance totale qui sont expliqués par chacun des facteurs.

\section{1. $p H$}

Il apparaît essentiellement influencé par les conditions d'affinage, les valeurs les plus élevées $(5,87-5,88)$ correspondant aux conditions extrêmes: affinage long à basse température (A1) et affinage à durée normale à $17,5^{\circ} \mathrm{C}$ (A3).

Les couples temps-température extrêmes mènent à des $\mathrm{pH}$ voisins malgré des états biochimiques de dégradation très différents, puisque nous verrons ultérieurement que les fromages de $\mathrm{A} 1$, possèdent un retard important au niveau de la protéolyse et de la fermentation propionique.

\section{TABLEAU II}

Résultats d'analyses de la variance des principales caractéristiques physico-chimiques des fromages au stade coupe : pourcentage de la variance expliqué par les différents facteurs

Results of variance analyses of the main physico-chemical characteristics of the cheese at the end of the ripening period: percent of the variances explained by the different factors

\begin{tabular}{|c|c|c|c|c|}
\hline \multirow[b]{2}{*}{$\begin{array}{l}\text { Variable } \\
\text { Variable }\end{array}$} & \multicolumn{3}{|c|}{ Facteurs (factors) } & \multirow[b]{2}{*}{$\begin{array}{l}\text { Total } \\
\text { Total }\end{array}$} \\
\hline & $\begin{array}{c}\text { Affinage } \\
\text { Ripening } \\
\text { conditions }\end{array}$ & $\begin{array}{l}\text { Fromagerie } \\
\text { Cheese plant }\end{array}$ & $\begin{array}{l}\text { Saison } \\
\text { Season }\end{array}$ & \\
\hline $\begin{array}{l}\mathrm{pH} \\
\mathrm{pH}\end{array}$ & $14,7 * *$ & $10,4 *$ & $4,0^{*}$ & 29,0 \\
\hline $\begin{array}{l}\text { Extrait sec } \\
\text { Dry matter }\end{array}$ & $31,7^{* *}$ & $8,1 *$ & $3,5 *$ & 43,3 \\
\hline $\begin{array}{l}\text { Calcium } \\
\text { Calcium }\end{array}$ & N.S. & $32,5 * *$ & $9,7^{* *}$ & 42,2 \\
\hline $\begin{array}{l}\text { Chlorure } \\
\text { de sodium } \\
\text { Sodium chloride }\end{array}$ & $14,3^{* *}$ & $36,6^{* *}$ & $5,4^{*}$ & 56,3 \\
\hline $\begin{array}{l}\text { Azote total } \\
\text { Total nitrogen }\end{array}$ & N.S. & $23,4 * *$ & $42,0 * *$ & 65,4 \\
\hline $\begin{array}{l}\text { Graisse } \\
\text { Fat }\end{array}$ & - & $12,1 * *$ & $37,7 * *$ & 49,8 \\
\hline \multicolumn{5}{|c|}{$\begin{array}{ll}\text { N.S. } & \text { : non significatif } \\
\text { non significant } \\
* & \text { : significatif } \mathrm{P}<0,05 \\
\text { significant } P<0.05 \\
{ }^{* *} & : \text { significatif } \mathrm{P}<0,01 \\
& \text { significant } P<0.01\end{array}$} \\
\hline
\end{tabular}




\section{Extrait sec}

Le calcul du gain d'extrait sec par unité de temps donne en valeur moyenne $1,41 \pm 0,52 \mathrm{~g} / 100 \mathrm{~g}$ de fromages par mois. Ce gain mensuel est très fortement corrélé à la température d'affinage $(\mathrm{r}=0,72)$ et à l'humidité relative des caves $(r=-0,58)$. Il n'est cependant pas possible de dissocier le rôle exact de la température d'affinage et de l'humidité relative des caves à partir du protocole expérimental. Le rôle de l'affinage est illustré également par une augmentation très nette de la variabilité de l'extrait sec des 96 fromages dont l'écart-type passe de $0,49 \mathrm{~g} / 100 \mathrm{~g}$ de fromage au stade $\mathrm{F}$ à $0,96 \mathrm{~g} / 100 \mathrm{~g}$ de fromage au stade $\mathrm{C}$.

L'influence de la fromagerie est faible mais significative. On constate une augmentaion de l'extrait sec moyen des fromages au cours de l'affinage qui va de $3,04 \mathrm{~g} / 100 \mathrm{~g}$ pour $\mathrm{F} 4$ à $3,81 \mathrm{~g} / 100 \mathrm{~g}$ pour $\mathrm{F} 1$. Ces différences ne semblent pas liées à l'extrait sec de départ mais plutôt à la taille moyenne des fromages; les fromages de F4 pesant en moyenne $43,40 \mathrm{~kg}$ au stade $\mathrm{F}$ contre $39,70 \mathrm{~kg}$ pour $\mathrm{F} 1$. Pour l'ensemble de la population des fromages, on a obtenu une corrélation faible mais significative de $-0,28$ entre la perte de poids (que l'on peut associer à une augmentation de l'extrait sec) et le poids des fromages. Par ailleurs, la perte de poids étant également corrélée avec l'humidité relative des caves $(\mathrm{r}=-0,51)$. Une étude de MuNCH (1983) montre l'intérêt économique de surveiller le couple humidité relative-température dans les caves d'affinage et préconise l'emploi d'une régulation assistée par microordinateur pour limiter les pertes de poids des fromages.

\section{Calcium}

- La fromagerie est le principal facteur de variation du taux de calcium des fromages. Ceci reflète un lien direct entre le taux de calcium des fromages d'une part, la richesse du lait en calcium, exprimée par le rapport $\mathrm{Ca}$ /caséine, et la perte de calcium liée à l'acidification du fromage sous presse d'autre part. En effet, pour les 24 fabrications nous avons obtenu un coefficient de corrélation significatif de $+0,39$ entre le taux calcium du fromage à 20 heures et le rapport $\mathrm{Ca}$ /caséine du lait, la corrélation passant à 0,56 lorsqu'on introduit dans le calcul de la corrélation multiple la teneur en lactate $\mathrm{D}$ du fromage à 20 heures.

Bien que les rapports $\mathrm{Ca} /$ caséine des laits soient très voisins pour les quatre fromageries (GRAPPIN et al., 1987), l'analyse de variance (tabl. 2), montre une différence significative dans les taux moyens des fromages affinés. La fromagerie $F 4$, qui possède les levains les moins acidifiants et pour laquelle on note un faible taux d'acide lactique D dans les fromages à 20 heures, présente la teneur en calcium la plus élevée.

- L'influence significative de la saison de fabrication sur le taux de calcium des fromages (tabl. 1 et 2 ) est le reflet de mécanismes indépendants. La teneur en calcium des fromages plus faible l'été (Grappin et al., 1987) s'explique en partie par un rapport $\mathrm{Ca} /$ caséine plus faible des laits.

- L'effet affinage n'apparaît pas significatif dans le cadre du plan factoriel de l'expérimentation. Cependant, si l'on crée 5 classes de teneur en 
ammoniac des caves, on observe alors une nette relation inverse entre les teneurs moyennes en ammoniac par classes et la teneur moyenne en calcium des fromages (fig. 2).

Une augmentation de la teneur en ammoniac de 24 à 65 ppm est liée à une faible décalcification de la pâte, le taux de calcium passant de 1,48 à 1,45 $\mathrm{g} / 100 \mathrm{~g}$ d'extrait sec, soit une baisse de $2,03 \%$ en valeur relative. Des essais réalisés sur camemberts placés en atmosphère ammoniacale ont montré une migration minérale qui entraîne une accumulation du phosphore et du calcium dans la croûte (LePIEnNe, 1982 ; Ribadeau-Dumas, 1984). Selon ces auteurs, l'atmosphère ammoniacale provoquerait une remontée chimique du $\mathrm{pH}$ dans la partie externe du fromage qui induirait une migration des solutés vers la surface, mais dans des proportions beaucoup plus importantes qu'en Comté.

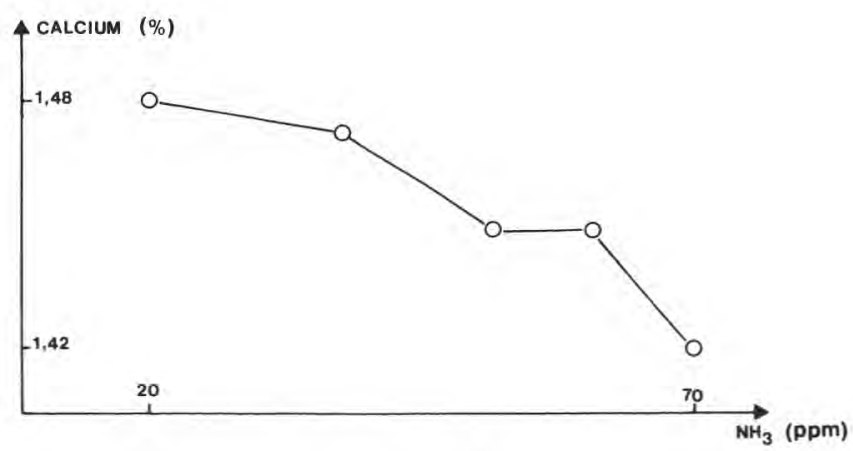

Fig. 2

Evolution moyenne de la teneur en calcium des fromages ( $g$ pour $100 \mathrm{~g}$ d'extrait sec) en fonction de la teneur en ammoniac (ppm) de l'atmosphère des caves d'affinage.

Mean evolution of the calcium content of the cheeses (in $\mathrm{g}$ per $100 \mathrm{~g}$ of dry matter) according to the ammoniac content (ppm) of the atmosphere of the ripening rooms.

\section{Chlorures de sodium}

Bien que les fromages affinés par A2 présentent une teneur en sel plus faible que ceux des autres affineurs (tabl. 1), c'est la fromagerie qui représente le facteur le plus important des variations du taux de sel. La figure 3 montre que la teneur finale en sel dépend essentiellement de la quantité de sel apportée à la fromagerie avant l'affinage en cave chaude. Les grains de sel lors de l'affinage ne sont pas identiques pour les quatre fromageries, c'est la fromagerie F4 qui par ailleurs présente le plus faible extrait sec $(63,27 \%)$ à l'entrée en cave d'affinage (stade A) contre 64,01 à 64,16 pour les trois autres fromageries, qui a le gain de sel le plus élevé au cours de l'affinage en cave chaude, soit $0,47 \mathrm{~g} / 100 \mathrm{~g}$ extrait sec contre $0,35,0,42$ et 0,35 pour $\mathrm{F} 1, \mathrm{~F} 2$ et 
F3. En revanche, bien qu'il existe une corrélation positive élevée entre la teneur en sel et l'extrait sec des fromages en fin d'affinage (LEFIER, 1986), aucun lien n'a pu être mis en évidence entre l'augmentation de l'extrait sec et le gain de chlorures en cave de fromagerie. Les conditions non standardisées de l'affinage en cave de fromagerie ayant probablement affaibli nos moyens d'observation.

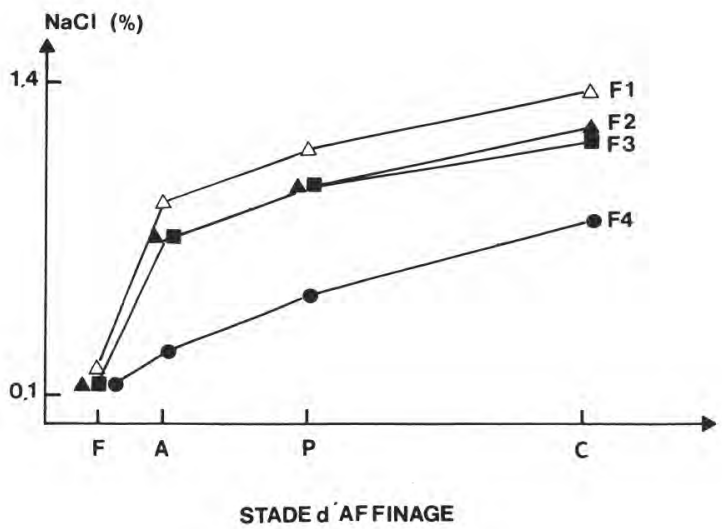

Fig 3.

Evolution de la teneur en chlorure de sodium des fromages ( $\mathrm{g}$ pour $100 \mathrm{~g}$ d'extrait sec) au cours de l'affinage en fonction de la fromagerie.

$F: 20 \mathrm{~h} ; A: 3$ semaines ; $P: 6$ semaines ; $C:$ fin d'affinage

Fromageries F1, F2, F3, F4.

Evolution of the sodium chloride content ( $\mathrm{g}$ per $100 \mathrm{~g}$ of dry matter) during ripening according to cheese plant.

$F: 20 h ; A: 3 w k ; P: 6 w k ; C:$ end of ripening

Cheese plant: F1, F2, F3, F4.

\section{Conclusion}

La plupart des caractéristiques physico-chimiques des fromages, dont l'importance est primordiale pour la qualité finale du produit, sont modifiées par les facteurs de l'étude.

Mis à part le gras/sec qui est uniquement lié à la technologie de fabrication et le taux de calcium partiellement influencé par la teneur en ammoniac de l'atmosphère des caves d'affinage qui favorise sa migration vers la croûte, les deux autres éléments, extrait sec et teneur en sel, sont influencés aussi bien par la technologie fromagère que par les conditions d'affinage qui comprennent l'affinage en cave froide à la fromagerie. 
Ainsi que cela avait déjà été souligné dans le premier article (Grappin et al., 1987), on note que les caractéristiques physico-chimiques de la fromagerie F4 se distinguent de celles des trois autres fromageries avec un gras/sec, un extrait $\mathrm{sec}$ et une teneur en sel plus faibles.

Reçu le 9 juillet 1986.

Accepté pour publication le 10 novembre 1986.

\section{Références bibliographiques}

Geurts T.J., Walstra P., Mulder H., 1974. Transport of salt and water during salting of cheese. 1. Analysis of the processes involved. Neth. Milk Dairy J., 28, 102-129.

Guerts T.J., Walstra P., Mulder H., 1980. Transport of salt and water during salting of cheese. 2. Quantities of salt taken up and of moisture lost. Neth. Milk Dairy J., 34, 229-254.

Grappin R., Berdague J.L., Dasen A., Jeunet R., 1987. Affinage et qualité du Gruyère de Comté. I. Protocole expérimental. Caractérisation des conditions d'affinage et des ateliers de fabrication. Lait, 67 (2) 219-235.

Heiss E., 1961. Essais de dosage de la matière grasse dans le fromage par des méthodes rapides. Dtsch. Molk. Ztg., 82, 3.

Lefier D., 1986. La lainure du Comté. Document non publié.

Le Graet Y., Lepienne A., Brule G., Ducruet P., 1983. Migration du calcium et des phosphates inorganiques dans les fromages à pâte molle de type Camembert au cours de l'affinage. Lait, $63,317-332$.

LEPIENNE A., 1982. Etude de la migration des éléments minéraux au cours de l'affinage des pâtes molles en atmosphère isolée. Mémoire de fin d'études. ENSA Rennes.

MAYR A., 1976. Zur Kochsaltzkonzentration in Emmentalerkäse. Dtsch. Molk. Ztg., 97, 493-496.

Metche M., Fanni J., 1978. Rôle de la flore fongique dans l'accumulation du calcium et du phosphore à la surface des fromages de type Camembert. Lait, 58, 336-354.

Munch P., 1983. Mesures de température et d'humidité relative dans les caves de fromagerie. Dtsch. Molk. Ztg., 104, 292-296.

PeArCE K.N., 1977. The complexometric determination of calcium in dairy products. N.Z.J. Dairy Sci. Technol., 12, 113-115.

Ribadeau-Dumas B., 1984. Maîtrise de l'affinage des fromages de type Camembert. Lait, 64, 448468. 